\title{
CASE REPORT \\ Efavirenz as a cause of ataxia in children
}

\author{
M P K Hauptfleisch, ${ }^{1}$ MB BCh, MMed (Paed), FCPaed (SA), Cert Paed Neuro (SA); \\ D P Moore, ${ }^{2}$ MB BCh, MMed (Paed), FCPaed (SA), Cert ID Paed (SA), MPhil (Paed ID); J L Rodda, ${ }^{1}$ MB BCh, FCPaed (SA) \\ ${ }^{1}$ Paediatric Neurology, Department of Paediatrics, Chris Hani Baragwanath Academic Hospital, University of the Witwatersrand, \\ Johannesburg, South Africa \\ ${ }^{2}$ Department of Paediatrics, Chris Hani Baragwanath Academic Hospital, University of the Witwatersrand, Johannesburg, South Africa
}

Corresponding author: M P K Hauptfleisch (marc.hauptfleisch@wits.ac.za)

\begin{abstract}
Acute ataxia in childhood is often caused by toxin ingestion. With the increasing number of paediatric patients on antiretroviral medication, we observe more side-effects of these drugs. Acute ataxia is defined as unsteadiness of walking or fine motor movement of $<72$ hours. The most common causes are postinfectious acute cerebellar ataxia, toxin ingestion and Guillain-Barré syndrome. However, the possibility of a mass lesion must always be excluded.

Reported neurological abnormalities in HIV-positive children range from $10 \%$ to $68 \%$. A South African study found the prevalence of neurological complications to be $59 \%$, the most common of which were HIV encephalopathy and long-tract motor signs; however, no cases of cerebellar dysfunction were documented. Ataxia rarely ocurs in an HIV-positive person, the chronic sequelae being neurocognitive impairment and polyneuropathy.

Ataxia in the setting of HIV is generally secondary to an infectious, vascular or neoplastic cerebellar lesion. However, most infections are opportunistic and unlikely to occur when CD4 levels are adequate. The vascular or mass lesions are readily excluded with neuro-imaging.

We report two cases of efavirenz toxicity that caused ataxia. We treated two children who presented in a 1-month period, which highlighted an important differential to consider in HIV-positive paediatric patients presenting with ataxia.

S Afr Med J 2015;105(10):876. DOI:10.7196/SAMJnew.8780
\end{abstract}

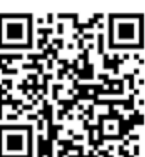

Acute ataxia in childhood is often caused by toxin ingestion. With the increasing number of paediatric patients on antiretroviral medication, we observe more side-effects of these drugs. We report two separate cases of efavirenz toxicity that caused ataxia.

\section{Background}

A child presenting with ataxia may pose a diagnostic dilemma. After excluding the common causes, e.g. toxins, infection and tumours, one needs to consider, more carefully, a possible genetic cause, but with limited genetic testing facilities available in South Africa (SA) a definitive answer is not always found.

It is estimated that there are $360000 \mathrm{HIV}$-positive children $<15$ years of age in $\mathrm{SA},{ }^{[1]} 44 \%$ of whom are on antiretroviral therapy (ART). ${ }^{[2]}$ These patients often pose unique diagnostic challenges.

We treated two children who presented in a 1-month period, which highlighted an important differential to consider in HIVpositive paediatric patients presenting with ataxia.

\section{Case 1}

A 6-year-old girl presented with acute onset of confusion, vomiting and difficulty walking. She was HIV-positive and had been on firstline ART (abacavir, lamivudine and efavirenz) for the past year. The antiretrovirals were dosed appropriately according to the child's weight and the Southern African HIV Clinicians Society guidelines. Her viral load 1 month before admission was $<100$ RNA copies/mL and her CD4 count 250 cells $/ \mu \mathrm{L}$.

There was no history of toxin ingestion.

On examination she was markedly ataxic, unable to walk or sit unsupported, and was noted to have titubation, dysmetria and dysdiadochokinesia.

Investigations for a possible infectious cause were done. The lumbar puncture (LP) was normal and serology for Varicella was negative; however, a CT scan of her brain was suggestive of possible cerebelitis. Although the cerebellar signs started acutely, they continued for $>2$ weeks. We therefore investigated further for possible inherited causes of progressive chronic ataxia. Our differential diagnosis included ataxia telangiectasia (no telangiectasia were present clinically and the immunoglobulin levels were normal), abetalipoproteinaemia, Friedreich's ataxia and spinocerebellar ataxia, but genetic testing was negative.

A magnetic resonance imaging (MRI) scan to better delineate the posterior fossa structures revealed a normal brain.

We considered medication toxicity because of the vomiting and the persistent ataxia. Therefore, an efavirenz level was determined and found to be $69100 \mathrm{ng} / \mathrm{mL}$ (target trough concentration: $1000 \mathrm{ng} / \mathrm{mL}$ ). On discontinuing the efavirenz the patient showed signs of clinical improvement, with resolution of the vomiting and ataxia. A protease inhibitor was not started as the elevated efavirenz levels were expected to act as the third antiretroviral agent.

A repeat efavirenz level 7 days after discontinuing the drug showed persistently high levels $(49000 \mathrm{ng} / \mathrm{mL}$ ), but the patient was able to walk unsupported and the dysmetria and dysdiadochokinesia had improved. We decided to administer lopinavir/ritonavir to the patient, and a month after discontinuing efavirenz she showed no residual cerebellar signs.

\section{Case 2}

A 13-year-old girl was referred to the paediatric neurology clinic with acute onset of an ataxic gait.

She was diagnosed with HIV 3 years previously and staged as World Health Organization clinical stage IV, but because of social issues was only started on ART (abacavir, lamivudine and efavirenz) 18 months before her referral to the neurology department. The doses of antiretrovirals were all appropriate for her weight, according to the Southern African HIV Clinicians Society guidelines. When she 
presented with ataxia she was virally suppressed, with an HIV viral load of 28 RNA copies $/ \mathrm{mL}$ and a CD4 count of 554 cells $/ \mu \mathrm{L}$.

On examination, she had a broad-based gait. Other cerebellar signs included dysmetria, dysdiadochokinesia and mild staccato speech, but no nystagmus.

Initial investigations were done to determine a possible infective cause. The LP was normal, and Varicella serology was negative. An MRI scan of her brain showed no intracranial lesions, structural changes or features of infection.

Before investigating further and because of our experience in the first case, we considered possible efavirenz toxicity - the level was $16274 \mathrm{ng} / \mathrm{mL}$.

Three weeks after discontinuing the drug, and relying on the elevated efavirenz levels to continue to act as the third antiretroviral agent, the efavirenz level had decreased to $1002 \mathrm{ng} / \mathrm{mL}$, with clinical improvement in her gait and resolution of her dysmetria. It was decided to opt for lopinavir/ritonavir as her third antiretroviral agent, and efavirenz was discontinued.

\section{Efavirenz drug level methodology in both cases}

Efavirenz is administered as a once-daily dose given at night to ameliorate its potential neurological side-effects. In both of the children reported here, drug levels were collected during the day, before $12 \mathrm{~h} 00$. Blood samples were collected in a $5 \mathrm{~mL}$ heparinised $\mathrm{BD}$ Vacutainer vial (BD Plymouth, UK). Specimens were stored at $2-8^{\circ} \mathrm{C}$ until analysed. Analysis was performed using liquid chromatographytandem mass spectrometry (LC-MS MS) at Ampath's laboratory in Johannesburg. An LC-MS MS TQD (triple quad detector) instrument from Waters in electrospray positive ionisation mode was used, employing an external standard from ChromSystems (Multilevel Plasma Calibrator Set Anti-HIV Drugs) for quantitation. Efavirenz is identified according to the molecular mass of its parent ion 316, as well as one daughter ion of molecular mass 244 , and retention time.

\section{Discussion}

Acute ataxia is defined as unsteadiness of walking or fine motor movement of $<72$ hours. The most common causes are postinfectious acute cerebellar ataxia, toxin ingestion and Guillain-Barré syndrome. ${ }^{[3]}$ However, the possibility of a mass lesion must always be excluded.

Reported neurological abnormalities in HIV-positive children range from $10 \%$ to $68 \%{ }^{[4]}$ An SA study found the prevalence of neurological complications to be $59 \%$, the most common of which were HIV encephalopathy and long-tract motor signs; however, no cases of cerebellar dysfunction were documented. ${ }^{[4]}$ The occurrence of ataxia in an HIV-positive person is rare, the chronic sequelae being neurocognitive impairment and polyneuropathy. ${ }^{[5]}$

Ataxia in the setting of HIV is generally secondary to an infectious, vascular or neoplastic cerebellar lesion. Most infections are opportunistic and unlikely to occur when the CD4 count is adequate. ${ }^{\left[{ }^{[j}\right.}$ The vascular or mass lesions are readily excluded with neuro-imaging.

Efavirenz is a non-nucleoside reverse transcriptase inhibitor that disrupts HIV replication by inhibiting the reverse transcriptase enzyme. Efavirenz is known to have good central nervous system (CNS) penetration ${ }^{[6]}$ and because of its long half-life is administered as a once-daily dose. The drug forms part of the SA first-line ART regimen for children $>3$ years of age and $>10 \mathrm{~kg}$ in weight. ${ }^{[7]}$

CNS symptoms are the most frequently reported side-effects in HIV-positive patients on efavirenz, including dizziness, headache, confusion, stupor, impaired concentration, agitation, amnesia, depersonalisation, hallucinations, insomnia and abnormal dreams. ${ }^{[6,8-14]}$
The frequency of CNS side-effects is estimated at $20-40 \%{ }^{[10]}$ The majority of patients develop CNS and psychiatric adverse effects in the first 6 weeks of treatment, ${ }^{[1]]}$ with most symptoms resolving 6 - 10 weeks after treatment initiation. ${ }^{[6]}$ Hoffmann et al ${ }^{[12]}$ documented neurocerebellar side-effects in patients using efavirenz. These symptoms were based on patient selfreported dizziness, ataxia, insomnia, bad dreams and hallucinations - there was no objective assessment of the ataxia. The neurocerebellar symptoms were again reported to occur most frequently in the first month of initiating efavirenz and declined with time. ${ }^{[12]}$

Many studies have examined the effect of efavirenz drug levels on the frequency of side-effects. Marzolini et al. ${ }^{[10]}$ reported a $24 \%$ increase in CNS side-effects if the plasma level was $>4000 \mathrm{ng} / \mathrm{mL}$; however, other research groups have found no correlation between adverse effects and plasma concentrations. ${ }^{[8,9,13]}$ Gutierrez et al. ${ }^{[13]}$ showed that CNS side-effects associated with long-term efavirenz administration were related to efavirenz plasma levels.

Wide variations in the plasma levels of patients on the same weight-appropriate dose of efavirenz have been demonstrated. One of the causes attributed to this individual variation in efavirenz levels is polymorphism of the CYP2B6 metabolising enzyme of the cytochrome P450, as efavirenz is a substrate of this enzyme. ${ }^{[9]}$ A study done in Botswana found that the prevalence of the slow metabolising genotype was $30 \%$, and other studies have also shown a higher incidence of this genotype in African populations. ${ }^{[14]}$

We conclude that the cause of ataxia in both our patients was attributable to the high plasma concentration of efavirenz. In both cases, the efavirenz level was at least 4 times greater than the toxic level $(4000 \mathrm{ng} / \mathrm{mL})$ described by Marzolini et al., ${ }^{[10]}$ and the ataxia improved when the drug was discontinued. Polymorphism of the CYP2B6 enzyme is a possibility in our patients, and may explain the very high plasma levels.

When presented with a child with acute progressive ataxia, who is known to be on efavirenz, and after excluding the common causes, one should check the efavirenz plasma levels and, if toxic, consider discontinuing the agent and substituting it with an alternative class of antiretroviral drug.

\section{References}

1. United Nations Programme on HIV/AIDS (UNAIDS). HIV and AIDS estimates 2012. http://www. unaids.org/en/regionscountries/countries/southafrica/ (accessed 15 January 2015).

2. United Nations Programme on HIV/AIDS (UNAIDS). The GAP report 2014. http://www.unaids. org/sites/default/files/en/media/unaids/contentassets/documents/unaidspublication/2014/UNAIDS _ Gap_report_en.pdf (15 January 2015).

3. Whelan HT, Verma S, Guo Y, et al. Evaluation of the child with acute ataxia: A systematic review. Welan HT, Verma S, Guo Y, et al. Evaluation of the child with acute ataxia: A systen
Pediatr Neurol 2013;49(1):15-24. [http://dx.doi.org/10.1016/.pediatrneurol.2012.12.005]

4. Govender R, Eley B, Walker K, Petersen R, Wilmshurst JM. Neurologic and neurobehavioral sequelae Govender R, Eley B, Walker K, Petersen R, Wilmshurst JM. Neurologic and neurobehavioral sequelae
in children with human immunodeficiency virus (HIV-1) infection. J Child Neurol 2011;26(11):1355in children with human immunodeficiency virus (HIV-1)

5. Anand KS, Wadhwa A, Garg J. A case of cerebellar ataxia associated with HIV infection. J Int Assoc Provid AIDS Care 2014;13(5):409-410. [http://dx.doi.org/10.1177/2325957414531620]

6. Treisman GJ, Kaplin AI. Neurologic and psychiatric complications of antiretroviral agents. AIDS 2002;16(9):1201-1215.

7. Health Systems Trust. National consolidated guidelines for the prevention of mother-to-child transmission of HIV (PMTCT) and the management of HIV in children, adolescents and adults 2014. http://www.hst.org.za/publications/national-consolidated-guidelines-prevention-mother-childtransmission-hiv-pmtct-and-man (accessed 15 January 2015).

8. Van Luin M, Bannister WP, Mocroft A, et al. Absence of a relation between efavirenz plasma concentrations and toxicity-driven efavirenz discontinuations in the EuroSIDA study. Antivir Ther 2009;14(1):75-83.

9. Takahashi M, Ibe S, Kudaka Y, et al. No observable correlation between central nervous system side effects and EFV plasma concentrations in Japanese HIV type 1-infected patients treated with EFV containing HAART. AIDS Res Hum Retrovir 2007;23(8):983-987.

10. Marzolini C, Telenti A, Decosterd LA, Greub G, Biollaz J, Buclin T. Efavirenz plasma levels can predict treatment failure and central nervous system side effects in HIV-1-infected patients. AIDS 2001;15(1):71-75.

11. Kappelhoff BS, van Leth F, Robinson PA, et al. Are adverse events of nevirapine and efavirenz related to plasma concentrations? Antivir Ther 2005;10(4):489-498.

2. Hoffmann CJ, Fielding KL, Charalambous S, et al. Antiretroviral therapy using zidovudine, lamivudine, and efavirenz in South Africa: Tolerability and clinical events. AIDS 2008;22(1):67-74.

3. Gutierrez F, Navarro A, Padilla S, et al. Prediction of neuropsychiatric adverse events associated with long-term efavirenz therapy, using plasma drug level monitoring. Clin Infect Dis 2005;41(11):16481653

14. Gross R, Aplenc R, Tenhave T, et al. Slow efavirenz metabolism genotype is common in Botswana. Acquir Immune Defic Syndr 2008;49(3):336-337. [http://dx.doi.org/10.1097/QAI.0b013e31817cled0] 